\title{
Humulus japonicus stimulates thermogenesis and ameliorates oxidative stress in mouse adipocytes
}

Tae Woo Jung1, Hyoung-Chun Kim², Yong Kyoo Shin¹, Hyeyoung Min³, Seong-Wan Cho4, Zi Soo Kim¹, Su Mi Han, A M Abd El-Aty5,6, Ahmet Hacımüftüoğlu' 6 and Ji Hoon Jeong1

'Department of Pharmacology, College of Medicine, Chung-Ang University, Seoul, Republic of Korea

${ }^{2}$ Neuropsychopharmacology and Toxicology Program, College of Pharmacy, Kangwon National University, Chunchon, Republic of Korea

${ }^{3}$ College of Pharmacy, Chung-Ang University, Seoul, Republic of Korea

${ }^{4}$ Department of Pharmaceutics \& Biotechnology, Konyang University, Daejeon, Republic of Korea

${ }^{5}$ Department of Pharmacology, Faculty of Veterinary Medicine, Cairo University, Giza, Egypt

${ }^{6}$ Department of Medical Pharmacology, Medical Faculty, Ataturk University, Erzurum, Turkey

Correspondence should be addressed to J H Jeong: jhjeong3@cau.ac.kr

\begin{abstract}
An aqueous extract of Humulus japonicus (AH) has been documented to ameliorate hypertension and non-alcoholic fatty liver disease (NAFLD). Here, we investigated the effects of an aqueous extract of $\mathrm{AH}$ on thermogenesis and palmitate-induced oxidative stress in adipocytes. To verify the effect of $\mathrm{AH}$ on browning, we measured the expression levels of specific markers in 3T3-L1 adipocytes using QPCR and Western blotting, respectively. To assess the role of oxidative stress, cells were stained with DCFDA and observed by fluorescence microscopy. AH increased the expression of brown adipose tissue-specific markers. Additionally, it induced fatty acid oxidation and lipolysis and suppressed both lipogenic markers and lipid accumulation. Furthermore, AH ameliorated hydrogen peroxide-induced oxidative stress. Enhanced expression of these markers contributed to fat browning, fatty acid oxidation and lipolysis of 3T3-L1 adipocytes via the AMP-activated protein kinase (AMPK) and peroxisome proliferator-activated receptor

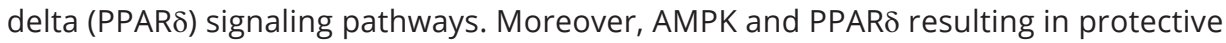
effects of AH against oxidative stress. In sum, AH could promote the browning, lipolysis and thermogenesis in 3T3-L1 adipocytes and would suppress the hydrogen peroxideinduced oxidative stress and lipogenesis during differentiation. We therefore suggest that $\mathrm{AH}$ could be used as a potential candidate for treating obesity and related metabolic disorders.
\end{abstract}

Journal of Molecular Endocrinology (2019) 63, 1-9

\section{Introduction}

Adipose tissue has multiple metabolic functions and plays a crucial role in homeostatic control of energy balance. Depending on the macroscopic appearance, adipose tissue has been featured to be white or brown. White adipose tissue (WAT) is composed of white adipocytes and primarily serve as an energy storage of neutral lipids. WAT stores excess energy for usage by other tissues during energy depletion. Brown adipose tissue (BAT) plays a prominent role in thermoregulation. In response to a variety of external conditions, cells strongly expressing 
uncoupling protein 1 (UCP1), which induced structural and functional changes in WAT to brown adipocytes (Lim et al. 2012). UCP1 dissipates the mitochondrial proton motive force and increases thermogenesis in BAT. Cold shock and $\beta 3$-adrenergic receptor activation by various external conditions may induce the appearance of brown adipocytes in WAT through remodeling of WAT adipocyte progenitors (Qiang et al. 2012). Recent advances in the treatment of obesity have revealed that WAT can demonstrate BAT-like phenotypes under certain external factors. Various pharmacological and dietary compounds have been suggested to increase energy expenditure and prevent lipid accumulation in mammals (Baboota et al. 2014, Roberts et al. 2014). For instance, capsaicin, $\beta$-isoaminobutyric acid (BAIBA) and irisin can stimulate the expression of browning-specific genes in adipocytes (Baboota et al. 2014, Roberts et al. 2014, Zhang et al. 2014a).

Owing to its multiple properties, Humulus japonicas has been reported to exert antimutagenic, antibacterial, antioxidant, anticancer and anti-inflammatory activities (Park et al. 1994, 1995, Hwang et al. 2009, Lee et al. 2012). It was reported that methanolic extract of $H$. japonicus could ameliorate atherosclerosis via suppression of the pro-atherogenic factors and lipid accumulation in the aortic endothelium of apolipoprotein E-deficient mice (Lim et al. 2016). Recently, the aqueous extract of $H$. japonicus (AH) was shown to alleviate hyperlipidemia and hepatic lipid accumulation in high-fat diet (HFD)-fed mice (Chung et al. 2018). However, the effects of AH on browning and oxidative stress in adipocytes have not yet been reported. Thus, in this study, we aimed to evaluate the effects of AH on stimulation of BAT-like phenotype in 3T3-L1 adipocytes. We further investigated the effects of $\mathrm{AH}$ on lipogenesis, lipolysis and oxidative stress and explored its molecular mechanisms.

\section{Materials and methods}

\section{Ethical approval}

This work does not contain any studies with human participants or animals performed by authors.

\section{Cell culture, reagents and antibodies}

We performed cell culture according to our previous protocol with modification (Jung et al. 2018a,b). Mouse preadipocytes (3T3-L1; ATCC) were cultured in Dulbecco's modified Eagle's medium (DMEM; Invitrogen) supplemented with $10 \%$ fetal bovine serum
(FBS) (Invitrogen), 100 U/mL penicillin and $100 \mu \mathrm{g} /$ $\mathrm{mL}$ streptomycin (Invitrogen). Cells were cultured in a humidified atmosphere containing $5 \% \mathrm{CO}_{2}$ at $37^{\circ} \mathrm{C}$. Two days after full confluence, cells were differentiated by incubation in DMEM containing insulin $(1 \mu \mathrm{M})$, IBMX $(0.5 \mathrm{mM})$ (Sigma), and dexamethasone $(0.5 \mu \mathrm{g} / \mathrm{mL})$ for 2 days and then in DMEM supplemented with insulin $(1 \mu \mathrm{M})$ for 3 days. Subsequently, cells were maintained in and refed every 3 days with a culture medium containing insulin $(1 \mu \mathrm{M})$ pending further experimental use (10 days after the initiation of differentiation). Differentiated 3T3-L1 cells were treated with AH $(0-100 \mu \mathrm{g} / \mathrm{mL}$ ) (I-woo Agricultural Company, Seoul, Republic of Korea) for $24 \mathrm{~h}$.

\section{Sample preparation}

Sample preparation was carried out according to our previous protocol with modification (Jung et al. 2018a,b). The lyophilized water extract was prepared using dried $H$. japonicus that was obtained from Woori Oriental Medicine Materials, and authenticated by Dr. Yuan Lu Sun of Solvit P\&F (Seoul, Republic of Korea). Dried H. japonicus $(650 \mathrm{~g})$ was mixed with distilled water $(15 \mathrm{~L})$ and boiled for $4 \mathrm{~h}$ at $100^{\circ} \mathrm{C}$ in duplicate. The extract was filtered, evaporated up to $15.6 \%$ and lyophilized using a lyophilizer (Labconco, Freezone 1L) at $5 \mathrm{mmHg}$ and $-50^{\circ} \mathrm{C}$. The lyophilized powder was stored at $-30^{\circ} \mathrm{C}$.

\section{Western blotting analysis}

Western blotting was performed according to (Jung et al. 2018a,b) with modification. Differentiated 3T3-L1 cells were harvested and total proteins were extracted with a lysis buffer (PRO-PREP; Intron Biotechnology, Seoul, Republic of Korea) for $60 \mathrm{~min}$ at $4^{\circ} \mathrm{C}$. Nuclear and cytosolic protein extracts were prepared using a protein fractionation kit (Biovision, Mountain View, CA, USA). Protein samples $(40 \mu \mathrm{g})$ were subjected to $12 \%$ SDS-PAGE, transferred to a nitrocellulose membrane (Amersham Bioscience, Westborough, MA, USA) and probed with the indicated primary antibodies followed by secondary antibodies conjugated with horseradish peroxidase (Santa Cruz Biotechnology). Signals were visualized using enhanced chemiluminescence (ECL) kits (Amersham Bioscience).

\section{RNA extraction and quantitative real-time PCR}

RNA extraction and quantitative real-time PCR were assayed according to the protocol of (Jung et al. 2018c) 
with modification. Total RNA was extracted from the harvested hepatocytes using TRIzol reagent (Invitrogen). Gene expression was measured by quantitative real-time PCR (qPCR) using the fluorescent TaqMan 5'nuclease assay on an Applied Biosystems 7000 sequence detection system (Foster City, CA, USA). qPCR was performed using cDNA as a template, $2 \times$ TaqMan Master Mix, and $20 \times$ premade TaqMan gene expression assays (Applied Biosystems). The qPCR conditions were as follows: $95^{\circ} \mathrm{C}$ for $10 \mathrm{~min}$, followed by 40 cycles of $95^{\circ} \mathrm{C}$ for $15 \mathrm{~s}$ and $60^{\circ} \mathrm{C}$ for $1 \mathrm{~min}$. PCR primer mixes for mouse $U c p$ 1 (Applied Biosystems; Mm01244861_m1), Pgc1 (Applied Biosystems; Mm01208832_m1), Prdm16 (Applied Biosystems; Mm00712556_m1), Cpt1 (Applied Biosystems; Mm01231183_m1), Aco (Applied Biosystems; Mm00801417_m1) and Fabp3 (Applied Biosystems; Mm02342495_m1) were used. The mRNA expression of $\beta$-actin was quantified as an endogenous control using the following primers: 5'-CGATGCTCCCCGGGCTGTAT-3' and 5'-TGGGGTACTTCAGGGTCAGG-3'.

\section{Transfection of cells with siRNAs for gene silencing}

siRNA transfection was conducted according to a previously modified protocol (Jung et al. 2018c). siRNA oligonucleotides $(20 \mathrm{nM})$ specific for AMP-activated protein kinase (AMPK) and peroxisome proliferatoractivated receptor delta (PPARס) were purchased from Santa Cruz Biotechnology. To suppress gene expression, cell transfection was performed using Lipofectamine ${ }^{\mathrm{TM}}$ 2000 (Invitrogen), according to the manufacturer's instructions. In brief, cells were grown to $60-70 \%$ confluence, followed by serum starvation for $12 \mathrm{~h}$ after 3T3-L1 cell differentiation. Cells were then transfected with validated siRNA or scramble siRNA at a final concentration of $20 \mathrm{nM}$ in the presence of the transfection reagent. Thirty-six hours post transfection, cells were harvested for protein extraction and additional analysis.

\section{Measurements of acetyl-CoA and ATP levels}

We measured acetyl-CoA and ATP content according to (Jung et al. 2018c) with modification. Intracellular levels of acetyl-CoA were measured in differentiated 3T3-L1 cells using a PicoProbe acetyl-CoA assay kit ${ }^{\mathrm{TM}}$ (Abcam), whereas the intracellular ATP levels were measured using an ATP assay $\mathrm{kit}^{\mathrm{TM}}$ (Abcam), according to the manufacturer's protocols.

\section{Oil red $\mathrm{O}$ staining and triglyceride (TG) assay}

Oil red O staining was performed according to the protocol described in our previous report with modification (Jung et al. 2018a,b). Differentiated 3T3-L1 cells were stained using Oil red $\mathrm{O}$ to measure the accumulated cellular neutral lipids, including TG. After fixation with $10 \%$ formalin for $40 \mathrm{~min}$, hepatocytes were stained with Oil red O solution (Sigma) for $1 \mathrm{~h}$ at $37^{\circ} \mathrm{C}$. Oil red O-stained TG was quantified by adding isopropanol to each sample (Yamaguchi et al. 2017). The mixtures were gently agitated at $25^{\circ} \mathrm{C}$ for $8 \mathrm{~min}$. Then, $100 \mu \mathrm{L}$ of isopropanol-extracted samples were analyzed using a spectrophotometer at $510 \mathrm{~nm}$.

\section{Determination of intracellular ROS accumulation}

We measured the cellular ROS levels according to the protocol modified from previous work (Jung et al. 2018a,b). Oxidative stress in adipocytes was evaluated using 5-(and 6)-chloromethyl-2', $7^{\prime}$-dichlorodihydrofluorescein

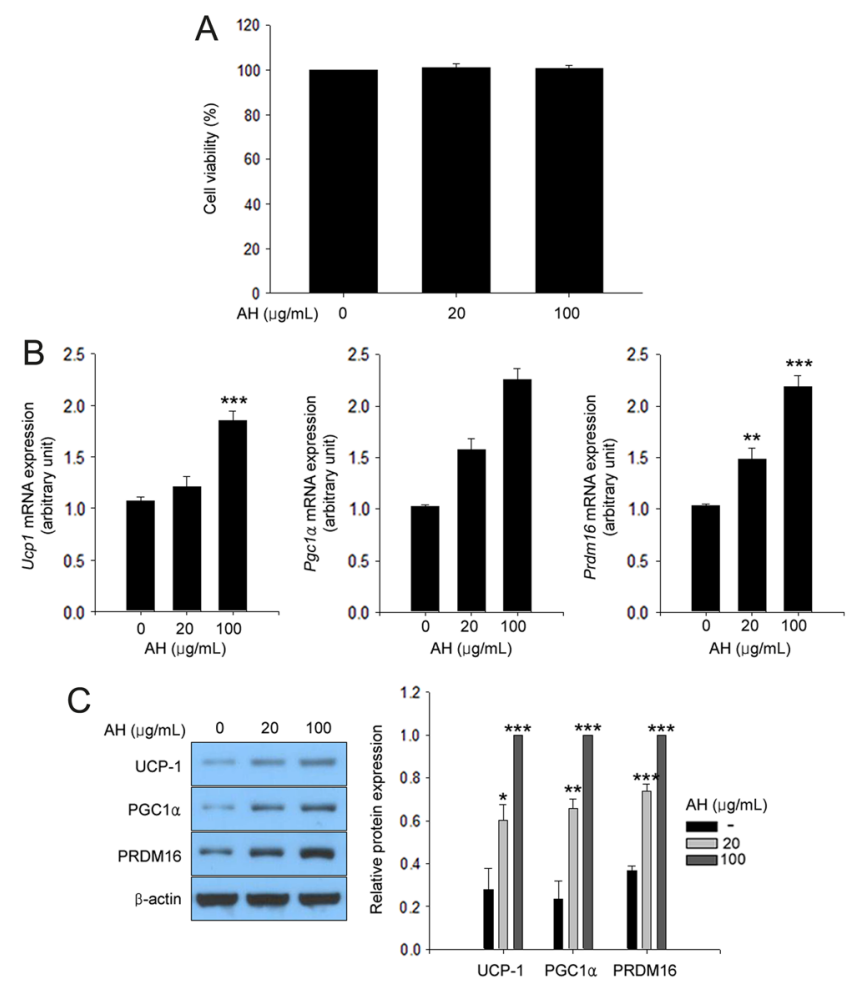

Figure 1

AH stimulates adipocyte browning. (A) Viability of 3T3-L1 adipocytes treated with $\mathrm{AH}$ at various concentrations $(0,20$ and $100 \mu \mathrm{g} / \mathrm{mL})$ for $24 \mathrm{~h}$ measured by MTT assay. (B) Quantitative real-time-PCR assay of Ucp1, Pgc1 $\alpha$ and Prdm16 in 3T3-L1 adipocytes treated with various concentrations of AH for $24 \mathrm{~h}$. (C) Western blotting analysis of UCP-1, PGC1 $\alpha$ and PRDM16 in 3T3-L1 adipocytes treated with various concentrations of AH for $24 \mathrm{~h}$. Mean \pm S.E.M. was obtained from three separate experiments. ${ }^{*} * P<0.001,{ }^{*} * P<0.01$ and $\star P<0.05$ compared to the controls. 
Figure 2
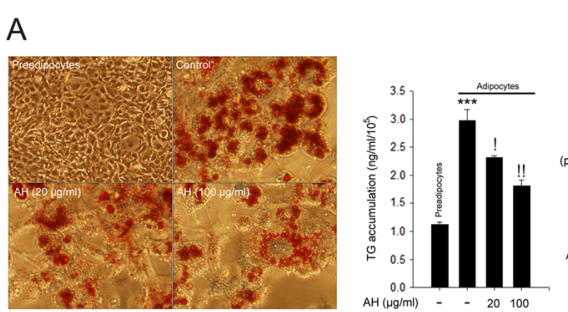

B
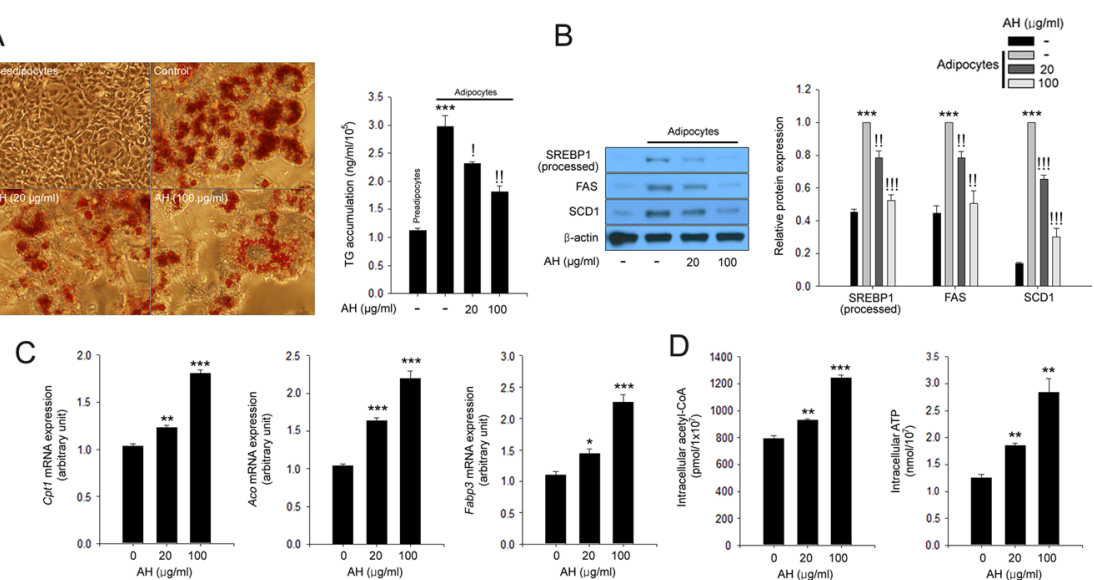

E

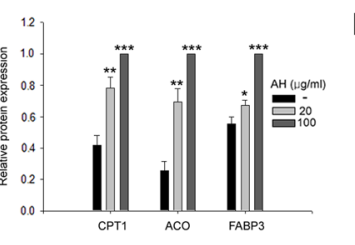

F

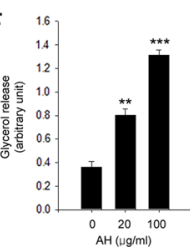

AH suppresses lipogenesis during differentiation and increases fatty acid oxidation and lipolysis in 3T3-L1 adipocytes. (A) Oil red $\mathrm{O}$ staining in preadipocytes and differentiated 3T3-L1 cells treated with $\mathrm{AH}(0,20$ and $100 \mu \mathrm{g} / \mathrm{mL})$ for $24 \mathrm{~h}$. Quantitative analysis of lipid accumulation was performed using an isopropanol extraction method. (B) Western blotting analysis of processed SREBP1, FAS and SCD1 in 3T3-L1 adipocytes treated with $\mathrm{AH}(0,20$ and $100 \mu \mathrm{g} / \mathrm{mL})$ for $24 \mathrm{~h}$. (C) Quantitative real-time-PCR assay of Cpt1, Aco and Fabp3 in 3T3-L1 adipocytes treated with $\mathrm{AH}(0,20$ and $100 \mu \mathrm{g} / \mathrm{mL})$ for $24 \mathrm{~h}$. (D) Intracellular acetyl Co-A and intracellular ATP levels were measured in 3T3-L1 adipocytes treated with $\mathrm{AH}(0,20$ and $100 \mu \mathrm{g} / \mathrm{mL})$ for $24 \mathrm{~h}$. Western blotting analysis of CPT1, ACO and FABP3 (E) and lipolysis ( $F$ ) in 3T3-L1 adipocytes treated with AH $(0,20$ and $100 \mu \mathrm{g} / \mathrm{mL})$ for 24 h. Mean \pm S.E.M. was calculated from data obtained from three independent experiments. $* \star \star P P<0.001, * \star P<0.01$ and $* P<0.05$ compared to controls. !! $P<0.001$, $! P<0.01$ and $! P<0.05$ compared to 3T3-L1 adipocytes. A full colour version of this figure is available at https://doi.org/10.1530/JME-19-0010. diacetate, acetyl ester (CM-H $\mathrm{H}_{2} \mathrm{DCFDA}$, Sigma). 3T3L1 adipocytes were treated with $5 \mu \mathrm{M}$ DCFDA for $1 \mathrm{~h}$. Following treatment, cells were stabilized by incubation with DMEM containing 10\% FBS for $10 \mathrm{~min}$. Cellular fluorescence levels were measured using a microplate reader set at an excitation wavelength of $485 \mathrm{~nm}$ and emission wavelength of $535 \mathrm{~nm}$.

\section{Dosage information}

Differentiated 3T3-L1 cells were treated with AH (0-100 $\mu \mathrm{g} /$ $\mathrm{mL}$ ) for $24 \mathrm{~h}$. Notably, fully differentiated 3T3-L1 cells did not show any sign of toxicity when treated with various concentrations of $\mathrm{AH}(0-100 \mu \mathrm{g} / \mathrm{mL})$ for $24 \mathrm{~h}$ or $48 \mathrm{~h}$.

\section{Statistical analysis}

Results were presented as absolute values (means \pm s.E.M.). All experiments were performed in triplicate. Data were analyzed using one-way ANOVA followed by Tukey's post hoc test. All analyses were performed using SPSS/PC (v 13.0 for Windows; SPSS).

\section{Results}

\section{AH increased the expression of BAT markers in 3T3-L1 adipocytes}

To investigate the effect of AH on the conversion of 3T3L1 adipocytes into brown adipocytes, we treated fully differentiated 3T3-L1 cells with various concentrations of $\mathrm{AH}(0-100 \mu \mathrm{g} / \mathrm{mL})$ for $48 \mathrm{~h}$ after the cell viability assay. AH treatment did not significantly affect cell viability at a concentration rate of $100 \mu \mathrm{g} / \mathrm{mL}$ (Fig. 1A). Furthermore, AH markedly increased the mRNA expression of BAT markers (Ucp1, PR domain containing 16 (PRDM16), and peroxisome proliferator-activated receptor gamma coactivator 1-alpha (PGC-1 $\alpha)$ ) in 3T3-L1 adipocytes in a dose-dependent fashion (Fig. 1B). Furthermore, treatment of 3T3-L1 adipocytes with $\mathrm{AH}$ increased the expression of UCP1, PRDM16 and PGC- $1 \alpha$ proteins in a dose-dependent manner (Fig. 1C).

\section{AH regulated fat metabolism in adipocytes}

As lipid metabolism plays a crucial role in browning in adipose tissue (Heeren \& Scheja 2018), we next examined whether AH could affect lipogenesis, fatty acid oxidation and lipolysis in 3T3-L1 adipocytes. Treatment of 3T3-L1 adipocytes with AH suppressed the expression of lipogenesis-associated enzymes (sterol regulatory element-binding protein 1 (SREBP1), FAS and stearoylCoA desaturase 1 (SCD1)) and decreased the lipid content (Fig. 2A and B), whereas it increased the expression of $\beta$-oxidation markers (carnitine palmitoyltransferase 1 (CPT1), ACO, and fatty acid-binding protein 3 (FABP3)), as well as cellular levels of acetyl-CoA and ATP (Fig. 2C, $\mathrm{D}$ and E). Additionally, AH treatment induced glycerol releases from differentiated 3T3-L1 cells in a dosedependent way (Fig. 2F), suggesting augmented lipolysis. 


\begin{tabular}{|l|l|l|l|l|}
$\begin{array}{l}\text { Journal of Molecular } \\
\text { Endocrinology }\end{array}$ & T W Jung et al. & $\begin{array}{l}\text { Humulus japonicus promotes } \\
\text { browning }\end{array}$ & $63: 1$ & $\mathbf{5}$ \\
\hline
\end{tabular}

A

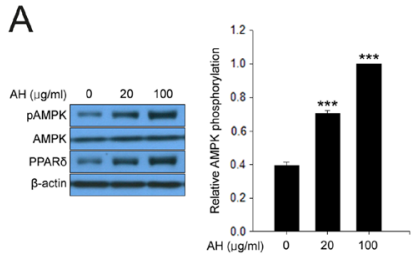

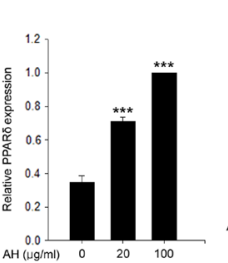

B
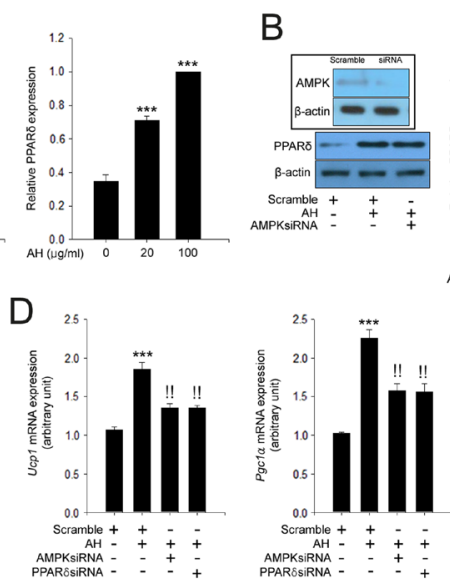
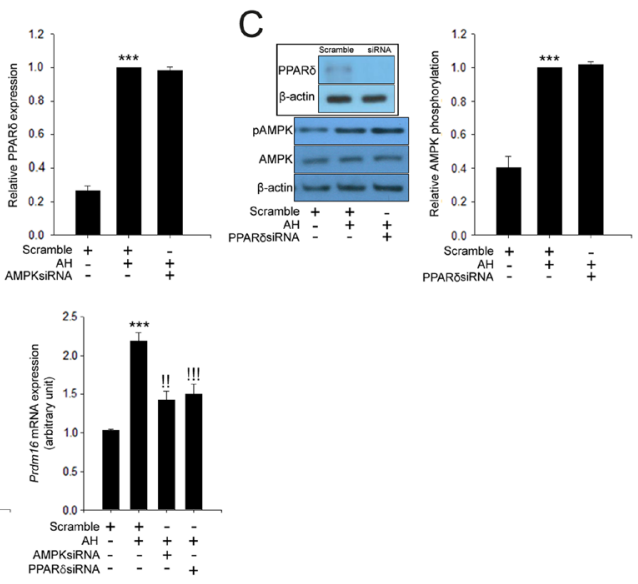

E
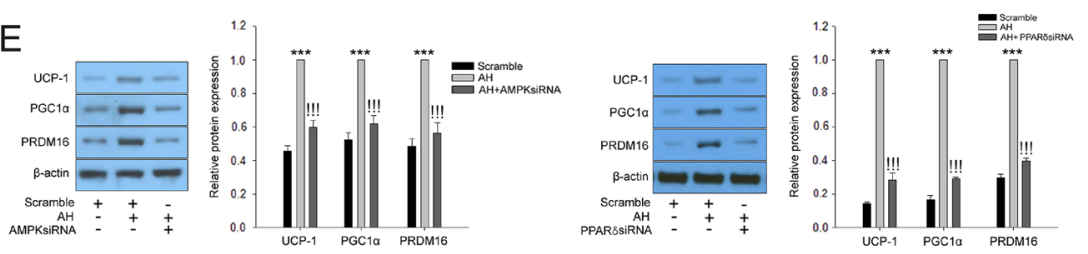

$\mathrm{F}$
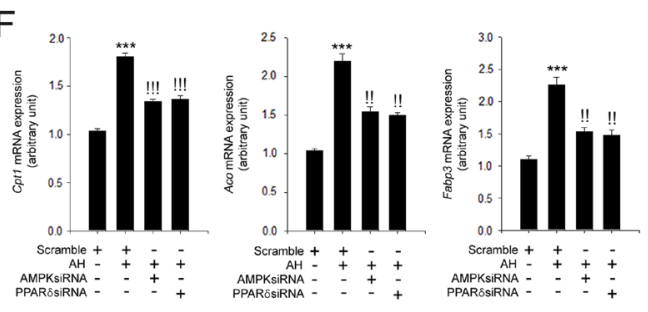

$\mathrm{G}_{1000}$
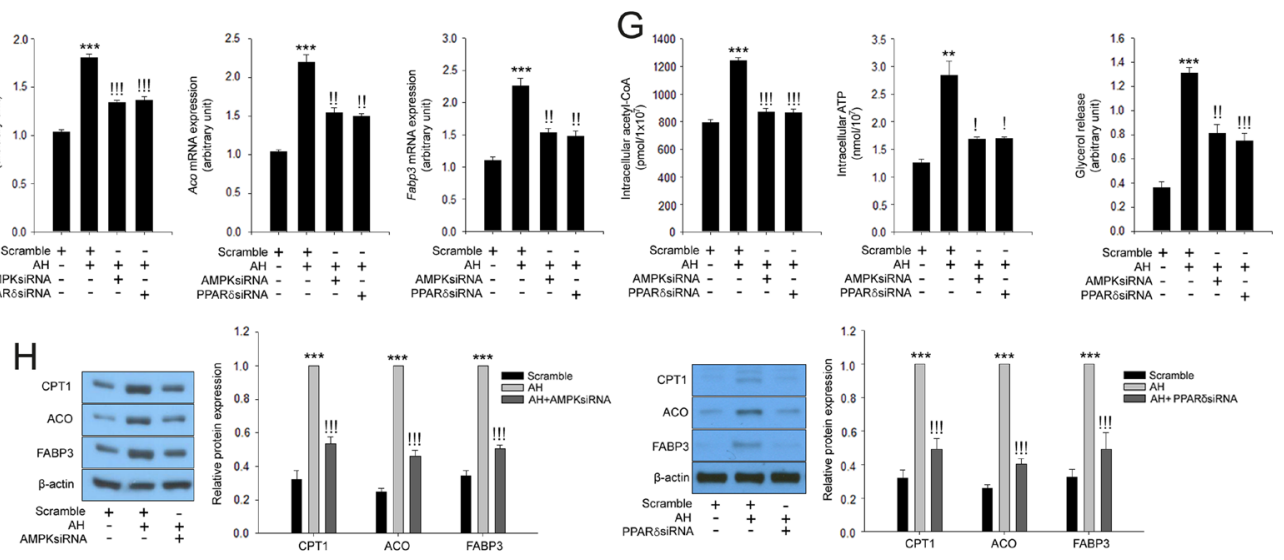

I
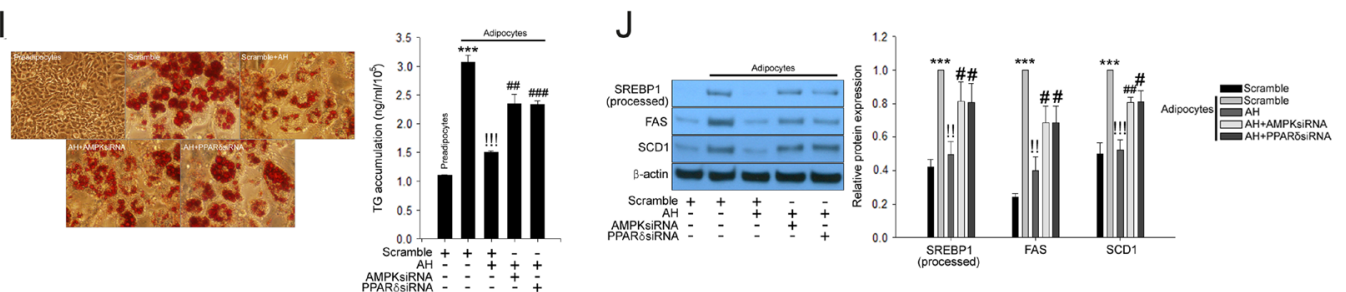

Figure 3

AH increases browning, fatty acid oxidation and lipolysis and suppresses lipogenesis during differentiation in 3T3-L1 adipocytes through AMPK or PPAR pathway. (A) Western blotting analysis of AMPK phosphorylation in 3T3-L1 adipocytes treated with AH (0,20 and $100 \mu \mathrm{g} / \mathrm{mL})$ for $24 \mathrm{~h}$. (B) Western blotting analysis of PPARS expression in scrambled or AMPKsiRNA-transfected 3T3-L1 adipocytes treated with AH for $24 \mathrm{~h}$. (C) Western blotting analysis of AMPK phosphorylation in scrambled or PPARסsiRNA-transfected 3T3-L1 adipocytes treated with AH for $24 \mathrm{~h}$. Quantitative real-time-PCR assay of Ucp1, Pgc1 $\alpha$ and Prdm16 (D) and Western blotting analysis of UCP1, PGC1 $\alpha$ and PRDM16 expression (E) in scrambled, AMPKsiRNA or PPARסsiRNA-transfected 3T3-L1 adipocytes treated with AH for $24 \mathrm{~h}$. Quantitative real-time-PCR assay of Cpt1, Aco and Fabp3 (F) and intracellular acetyl Co-A and intracellular ATP levels (G) were measured in scrambled, AMPKsiRNA or PPARסsiRNA-transfected 3T3-L1 adipocytes treated with AH for $24 \mathrm{~h}$. (H) Western blotting analysis of CPT1, ACO and FABP3 expression in scrambled, AMPKsiRNA or PPAR8siRNA-transfected 3T3-L1 adipocytes treated with AH for $24 \mathrm{~h}$. (I) Oil red O staining in preadipocytes and differentiated 3T3-L1 cells scrambled, AMPKsiRNA or PPARסsiRNA-transfected 3T3-L1 adipocytes treated with AH for $24 \mathrm{~h}$. Quantitative analysis of lipid accumulation was performed using an isopropanol extraction method. (J) Western blotting analysis of processed SREBP1, FAS and SCD1 in preadipocytes and differentiated 3T3-L1 cells scrambled, AMPKsiRNA or PPARסsiRNA-transfected 3T3-L1 adipocytes treated with AH for 24 h. Mean \pm S.E.M. was calculated from data obtained from three independent experiments. ${ }^{* \star *} P<0.001$ and $* \star P<0.01$ compared to controls. !"!P<0 0.001 , $! P<0.01$, and $! P<0.05$ compared to AH treatment in 3T3-L1 adipocytes or control in 3T3-L1 adipocytes. \#\#P<0.01 and $\# P<0.05$ compared to controls in 3T3-L1 adipocytes. A full colour version of this figure is available at https://doi.org/10.1530/JME-19-0010. 
A
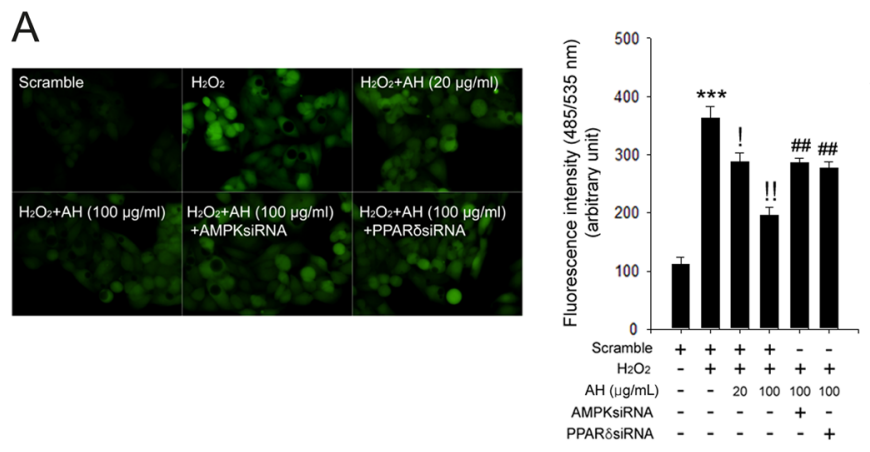

C
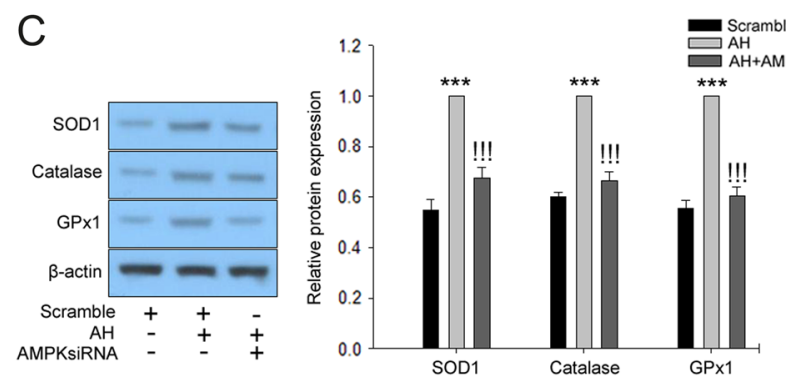

B
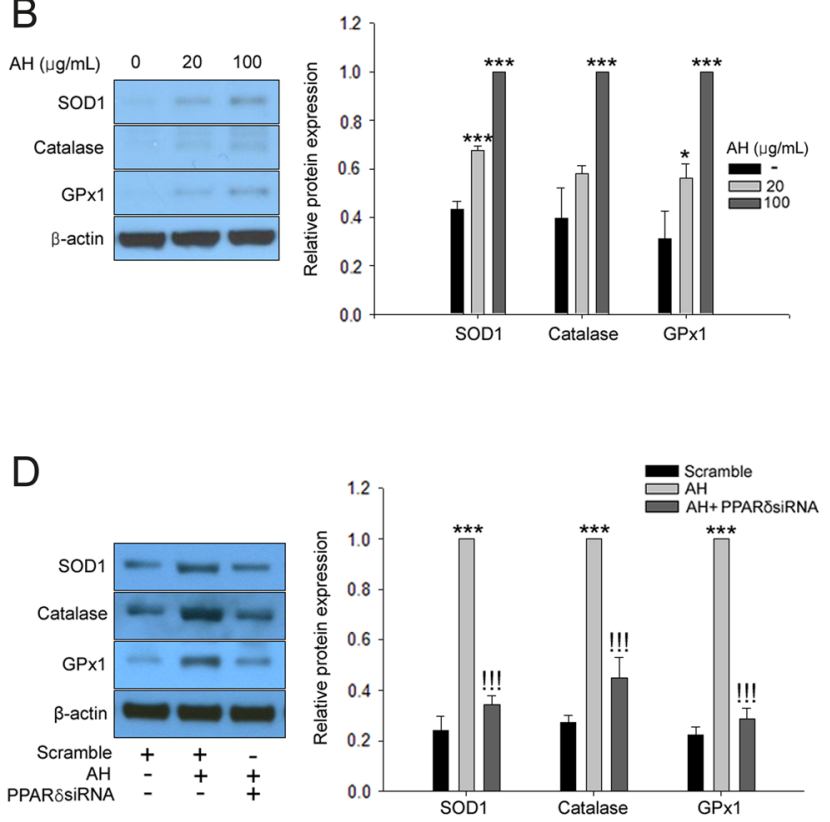

Figure 4

AH ameliorates oxidative stress through AMPK or PPAR8-mediated pathway. (A) ROS staining of preadipocytes and differentiated 3T3-L1 cells scrambled, AMPKsiRNA or PPARסsiRNA-transfected 3T3-L1 adipocytes treated with AH for $24 \mathrm{~h}$. Western blotting analysis of SOD1, catalase and GPx-1 expression in preadipocytes and differentiated 3T3-L1 cells in the presence of AH (0,20 and $100 \mu \mathrm{g} / \mathrm{mL})(B)$ and in preadipocytes and differentiated 3T3-L1 cells scrambled, AMPKsiRNA or PPARסsiRNA-transfected 3T3-L1 adipocytes treated with AH for $24 \mathrm{~h}$ (C and D). Mean \pm S.E.M. was calculated from data obtained from three independent experiments. $* \star \star P<0.001$ and $* P<0.05$ compared to controls. !! $P<0.001$, ! $P<0.01$ and ! $P<0.05$ compared to AH treatment or $\mathrm{H}_{2} \mathrm{O}_{2}$ treatment. \#\# $P<0.01$ compared to $\mathrm{H}_{2} \mathrm{O}_{2}$ and $\mathrm{AH}$ treatment in 3T3-L1 adipocytes. A full colour version of this figure is available at https://doi. org/10.1530/JME-19-0010.

\section{AH stimulated browning and $\beta$-oxidation via AMPK and PPARS-mediated pathways}

AMPK (Daval et al. 2006) and PPARס (Goto et al. 2011) have been reported to stimulate fatty acid oxidation. Therefore, to identify the possible mechanisms associated with AH-mediated 3T3-L1 adipocyte browning, we therefore investigated the key lipid metabolic regulators - AMPK and PPARס. Treatment of 3T3-L1 adipocytes with AH augmented AMPK phosphorylation and PPAR $\delta$ expression in a dosedependent manner (Fig. 3A). Interestingly, increased AMPK phosphorylation and PPAR $\delta$ expression by AH did not affect the activity or expression of each other (Fig. 3B and C). Therefore, we hypothesized that AMPK and PPAR $\delta$ might cause the induction of browning. Thence, we further investigated the effects of AMPK and PPARס suppression on AH-induced BAT markers. siRNAs of AMPK and PPARS mitigated the effects of AH on mRNA and protein expression of UCP1, PRDM16 and PGC1 $\alpha$ in 3T3-L1 adipocytes (Fig. 3D and E). Moreover, siRNA against AMPK and PPARס reversed AH effects on fatty acid oxidation markers, cellular acetyl-CoA, ATP contents and lipolysis (Fig. 3F, G and H). Furthermore,
AMPK and PPARס siRNA mitigated the effects of AH on lipid accumulation and expression of lipogenic proteins, such as processed SREBP1, FAS and SCD1 (Fig. 3I and J).

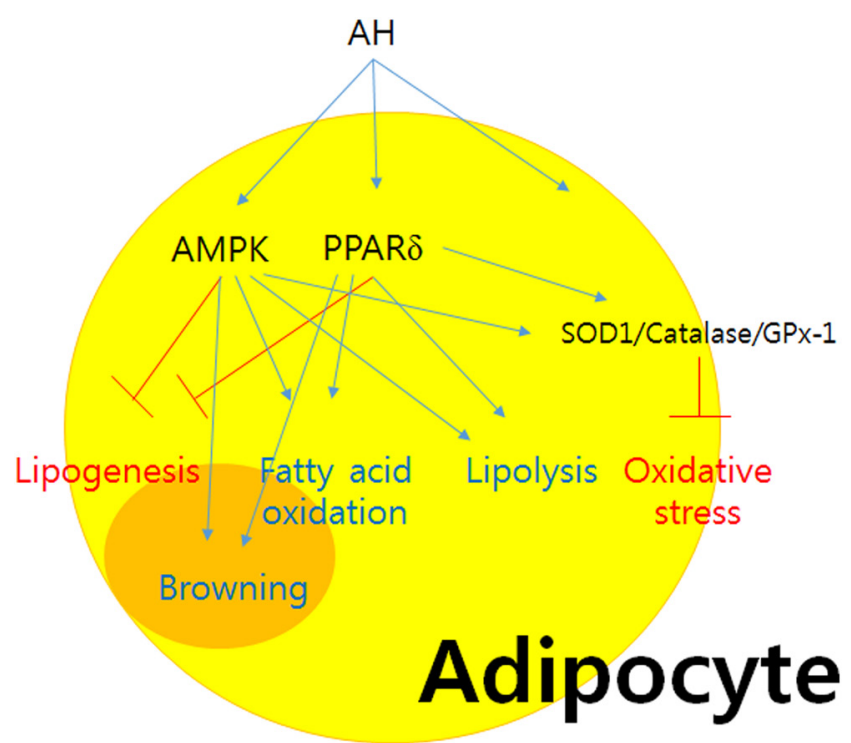

Figure 5

Schematic diagram of the effects of AH on adipocytes. A full colour version of this figure is available at https://doi.org/10.1530/JME-19-0010. 


\section{AH attenuated hydrogen peroxide-induced oxidative stress in differentiated 3T3-L1 cells}

It was reported that oxidative stress could contribute to the development of obesity-associated metabolic disorders (Roberts \& Sindhu 2009). Therefore, we evaluated the effects of $\mathrm{AH}$ on oxidative stress. $\mathrm{AH}$ ameliorated hydrogen peroxide $\left(\mathrm{H}_{2} \mathrm{O}_{2}\right)$-induced ROS production in a dose-dependent fashion. Furthermore, siRNA against AMPK and PPAR $\delta$ weakened the inhibitory effects of AH on $\mathrm{H}_{2} \mathrm{O}_{2}$-induced ROS production (Fig. 4A). Treatment of 3T3-L1 cells with AH significantly increased the expression of antioxidant enzymes, such as superoxide dismutase 1 (SOD1), catalase and glutathione peroxidase 1 (GPx1) (Fig. 4B). However, siRNA against AMPK and PPARS reversed these changes (Fig. 4C and D).

\section{Discussion}

Conversion of WAT into BAT as an energy consumption strategy is a promising approach for treating obesity and other metabolic diseases (Tseng et al. 2010). In the same context, improving thermogenesis in BAT or brown adipocyte-like features in WAT can be a crucial therapeutic approach for treating obesity and related diseases (Kiefer et al. 2012). Generally, 3T3-L1 adipocytes have been used as white adipocytes in in vitro model (Morrison \& McGee 2015). In the present study, we verified that $\mathrm{AH}$ has the potential to stimulate brown adipocyte-like phenotype and ameliorate oxidative stress in fully differentiated 3T3L1 adipocytes. Although Chung et al. have reported that $\mathrm{AH}$ can ameliorate hyperlipidemia and non-alcoholic fatty liver through regulation of lipogenesis (Chung et al. 2018), it remains unclear how AH ameliorates obesity. In this study, treatment with AH significantly augmented UCP1, PRDM16 and PGC-1 $\alpha$ expression. These results suggest a possible role of $\mathrm{AH}$ in stimulating thermogenesis and browning, since UCP1, PRDM16 and PGC- $1 \alpha$ have been shown to contribute to the recruitment of beige cells to WAT. It has been documented that SREBP1, a crucial transcription factor for lipogenesis, plays a pivotal role in lipid metabolism (Ito et al. 2013). FAS and SCD1 are representative lipogenesis-associated genes. FAS regulates the de novo biosynthesis of long-chain fatty acids (Wakil 1989), whereas CD1 contributes to the biosynthesis of monounsaturated fatty acids, the precursors of triglycerides (Xu et al. 2015). Herein, we elucidated that AH treatment mitigated lipogenesis through inhibition of processed SREBP1, FAS and SCD1 expression. AH treatment also abrogated lipid accumulation. Hence, lipogenesis and lipid accumulation (common features of obesity) were suppressed in adipocytes treated with AH. It has been verified that CPT1, ACO and FABP3 play critical roles in fatty acid oxidation. Here, AH treatment increased the mRNA expression of these three genes in adipocytes. Furthermore, the cellular levels of acetyl-CoA and ATP, products of fatty acid oxidation, were also increased following AH treatment. These results showed that $\mathrm{AH}$ stimulated fatty acid oxidation in adipocytes; the finding which may indicate that $\mathrm{AH}$ could downsize adipocytes via suppression of lipogenesis, as well as induction of thermogenesis and fatty acid oxidation.

AMPK is a key metabolic regulator for maintaining cellular energy homeostasis (Hardie et al. 2012), by controlling catabolic and anabolic pathways (Zhang et al. 2009). AMPK plays a crucial role in various biological processes, such as cell survival, cell migration, autophagy and energy metabolism (Mihaylova \& Shaw 2011). Notably, AMPK plays a central role in the interplay between fatty acid metabolism and thermogenesis in adipose tissues (Day et al. 2017). It was reported that activation of AMPK stimulates fatty acid oxidation and suppresses fatty acid synthesis (Carlson \& Kim 1973, Fullerton et al. 2013). Moreover, AMPK regulates the biogenesis of mitochondria via activation of the PGC1 $\alpha$-mediated pathway (Jager $e t$ al. 2007). AMPK activation promotes thermogenesis in BAT and WAT through PGC1 $\alpha$-dependent signaling (Zhang et al. 2014b) and suppresses white adipocyte differentiation (Zhou et al. 2009). Therefore, it is necessitated to explore the molecular mechanisms, by which AH-mediated browning in white adipocytes. We have found that treatment with AH significantly augmented AMPK phosphorylation in differentiated 3T3-L1 cells. Moreover, AMPK inhibition mitigated the effects of $\mathrm{AH}$ on thermogenesis, fatty acid oxidation and lipolysis. These results suggested that AMPK might play a significant role in AH-mediated stimulation of adipocyte browning and anti-obesity effects.

PPAR belongs to the PPAR subfamily that also includes PPAR $\alpha$ and PPAR $\gamma$ (Desvergne \& Wahli 1999). These receptors regulate transcription through specific binding to PPAR response element (PPRE) in the promoter of target genes (Mangelsdorf et al. 1995). PPARs control diverse metabolic processes and activated by fatty acids (Lee et al. 1995). It has to be noted that PPARס is highly expressed in various metabolically active tissues. A previous study suggested that dietary fatty acids could regulate the expression of adipose differentiationrelated protein (ADRP), a lipid-coating protein, by very low-density lipoproteins through the PPARס-mediated pathway (Chawla et al. 2003). PPARס activation increased 
the expression of fatty acid oxidation-related genes (CPT1, ACO and long-chain acyl-CoA dehydrogenase (LCAD)), as well as thermogenic genes (UCP-1 and UCP-3) in BAT (Reilly \& Lee 2008). Yu et al. showed that fish oil could stimulate lipolysis via PPARס-dependent signaling (Yu et al. 2010). These studies provide evidence that PPAR $\delta$ plays an important role in lipid metabolism and could be a potential candidate for targeting metabolic disorders. In the present study, AH treatment markedly induced PPAR $\delta$ expression in 3T3-L1 adipocytes. Hence, suppression of PPAR $\delta$ mitigated AH-induced thermogenesis, fatty acid oxidation and lipolysis. These results suggest that AH stimulated adipocyte browning through the PPARסmediated pathway.

Furthermore, current data indicated that $\mathrm{AH}$ ameliorated oxidative stress in 3T3-L1 adipocytes. Elevated oxidative stress observed in obese patients has been suggested to contribute to the development of metabolic syndrome, such as obesity, insulin resistance, hypertension and dyslipidemia (Roberts \& Sindhu 2009). Increased levels of ROS in adipocytes might be attributed to reduced antioxidant enzymes, such as SOD, catalase and GPX (Furukawa et al. 2004). Therefore, AH can be used as a therapeutic approach for treating metabolic diseases via attenuating redox potential in WAT.

In conclusion, our results indicated that AH may play various roles in the stimulation of browning and lipid metabolism, and attenuation of oxidative stress in white adipocytes through AMPK or PPAR $\delta$-dependent pathways (Fig. 5). AH may constitute a new therapeutic strategy for the treatment of metabolic complications of obesity.

\section{Declaration of interest}

The authors declare that there is no conflict of interest that could be perceived as prejudicing the impartiality of the research reported.

\section{Funding}

This study was supported by the Basic Science Research Program through the National Research Foundation of Korea (NRF) funded by the Ministry of Science, ICT \& Future Planning (2016R1C1B2012674), the Korean Government Ministry of Education (2017R1D1A1B03028892) and ChungAng Academy Program 2017.

\section{Author contribution statement}

T W J, H C K, A A E, Y K S, H Y M, S W C, Z S K, S M H and J H J: substantial contribution to the conception and design; $\mathrm{T} W \mathrm{~J}$ and $\mathrm{J} \mathrm{H} \mathrm{J:} \mathrm{acquisition} \mathrm{of}$ data or analysis and interpretation of data; $\mathrm{H} \mathrm{CK}$ and A M A: drafting and revising the MS. All authors approved the final version of the manuscript. $\mathrm{T} \mathrm{W} J$ and $\mathrm{J} \mathrm{H} \mathrm{J} \mathrm{are} \mathrm{responsible} \mathrm{for} \mathrm{the} \mathrm{integrity} \mathrm{of} \mathrm{the} \mathrm{work} \mathrm{as} \mathrm{a} \mathrm{whole.}$

\section{References}

Baboota RK, Singh DP, Sarma SM, Kaur J, Sandhir R, Boparai RK, Kondepudi KK \& Bishnoi M 2014 Capsaicin induces "Brite" phenotype in differentiating 3T3-L1 preadipocytes. PLOS ONE 9 e103093. (https://doi.org/10.1371/journal.pone.0103093)

Carlson CA \& Kim KH 1973 Regulation of hepatic acetyl coenzyme A carboxylase by phosphorylation and dephosphorylation. Journal of Biological Chemistry 248 378-380. (https://doi.org/10.1016/00039861(74)90058-7)

Chawla A, Lee CH, Barak Y, He W, Rosenfeld J, Liao D, Han J, Kang H \& Evans RM 2003 PPARdelta is a very low-density lipoprotein sensor in macrophages. PNAS 100 1268-1273. (https://doi.org/10.1073/ pnas.0337331100)

Chung YH, Bang JS, Kang CM, Goh JW, Lee HS, Hong SM, Kim DS, Park ES, Jung TW, Shin YK, et al. 2018 Aqueous extract of Humulus japonicus attenuates hyperlipidemia and fatty liver in obese mice. Journal of Medicinal Food 21 999-1008. (https://doi.org/10.1089/ jmf.2017.4135)

Daval M, Foufelle F \& Ferre P 2006 Functions of AMP-activated protein kinase in adipose tissue. Journal of Physiology $\mathbf{5 7 4} 55-62$. (https://doi. org/10.1113/jphysiol.2006.111484)

Day EA, Ford RJ \& Steinberg GR 2017 AMPK as a therapeutic target for treating metabolic diseases. Trends in Endocrinology and Metabolism $\mathbf{2 8}$ 545-560. (https://doi.org/10.1016/j.tem.2017.05.004)

Desvergne B \& Wahli W 1999 Peroxisome proliferator-activated receptors: nuclear control of metabolism. Endocrine Reviews 20 649-688. (https://doi.org/10.1210/edrv.20.5.0380)

Fullerton MD, Galic S, Marcinko K, Sikkema S, Pulinilkunnil T, Chen ZP, O'Neill HM, Ford RJ, Palanivel R, O'Brien M, et al. 2013 Single phosphorylation sites in Acc1 and Acc2 regulate lipid homeostasis and the insulin-sensitizing effects of metformin. Nature Medicine 19 1649-1654. (https://doi.org/10.1038/nm.3372)

Furukawa S, Fujita T, Shimabukuro M, Iwaki M, Yamada Y, Nakajima Y, Nakayama O, Makishima M, Matsuda M \& Shimomura I 2004 Increased oxidative stress in obesity and its impact on metabolic syndrome. Journal of Clinical Investigation 114 1752-1761. (https:// doi.org/10.1172/JCI21625)

Goto T, Lee JY, Teraminami A, Kim YI, Hirai S, Uemura T, Inoue H, Takahashi N \& Kawada T 2011 Activation of peroxisome proliferatoractivated receptor-alpha stimulates both differentiation and fatty acid oxidation in adipocytes. Journal of Lipid Research 52 873-884. (https://doi.org/10.1194/jlr.M011320)

Hardie DG, Ross FA \& Hawley SA 2012 AMPK: a nutrient and energy sensor that maintains energy homeostasis. Nature Reviews: Molecular Cell Biology 13 251-262. (https://doi.org/10.1038/nrm3311)

Heeren J \& Scheja L 2018 Brown adipose tissue and lipid metabolism. Current Opinion in Lipidology 29 180-185. (https://doi.org/10.1097/ MOL.0000000000000504)

Hwang SY, Jung HJ, Jang WS, Jo MJ, Kim SC \& Jee SY 2009 Antiinflammaory effects of the $\mathrm{MeOH}$ extract of Humulus japonicus in vitro. Korean Oriental Medical Ophthalmology and Otolaryngology and Dermatology Society 22 71-79 [In Korean].

Ito M, Nagasawa M, Omae N, Tsunoda M, Ishiyama J, Ide T, Akasaka Y \& Murakami K 2013 A novel JNK2/SREBP-1c pathway involved in insulin-induced fatty acid synthesis in human adipocytes. Journal of Lipid Research 54 1531-1540. (https://doi.org/10.1194/jlr.M031591)

Jager S, Handschin C, St-Pierre J \& Spiegelman BM 2007 AMP-activated protein kinase (AMPK) action in skeletal muscle via direct phosphorylation of PGC-1alpha. PNAS 104 12017-12022. (https:// doi.org/10.1073/pnas.0705070104)

Jung TW, Chung YH, Kim HC, Abd El-Aty AM \& Jeong JH 2018a Protectin DX attenuates LPS-induced inflammation and insulin resistance in adipocytes via AMPK-mediated suppression of the NF-kappaB pathway. American Journal of Physiology: Endocrinology and https://jme.bioscientifica.com

https://doi.org/10.1530/JME-19-0010 (c) 2019 Society for Endocrinology Published by Bioscientifica Ltd. Printed in Great Britain 
Metabolism 315 E543-E551. (https://doi.org/10.1152/ ajpendo.00408.2017)

Jung TW, Kang C, Goh J, Chae SI, Kim HC, Lee TJ, Abd El-Aty AM \& Jeong JH 2018b WISP1 promotes non-alcoholic fatty liver disease and skeletal muscle insulin resistance via TLR4/JNK signaling. Journal of Cellular Physiology 233 6077-6087. (https://doi.org/10.1002/ jcp.26449)

Jung TW, Lee SH, Kim HC, Bang JS, Abd El-Aty AM, Hacimuftuoglu A, Shin YK \& Jeong JH 2018c METRNL attenuates lipid-induced inflammation and insulin resistance via AMPK or PPARdelta-dependent pathways in skeletal muscle of mice. Experimental and Molecular Medicine 50 122. (https://doi.org/10.1038/s12276-018-0147-5)

Kiefer FW, Vernochet C, O'Brien P, Spoerl S, Brown JD, Nallamshetty S, Zeyda M, Stulnig TM, Cohen DE, Kahn CR, et al. 2012 Retinaldehyde dehydrogenase 1 regulates a thermogenic program in white adipose tissue. Nature Medicine 18 918-925. (https://doi.org/10.1038/nm.2757)

Lee SS, Pineau T, Drago J, Lee EJ, Owens JW, Kroetz DL, FernandezSalguero PM, Westphal H \& Gonzalez FJ 1995 Targeted disruption of the alpha isoform of the peroxisome proliferator-activated receptor gene in mice results in abolishment of the pleiotropic effects of peroxisome proliferators. Molecular and Cellular Biology $\mathbf{1 5}$ 3012-3022. (https://doi.org/10.1128/MCB.15.6.3012)

Lee YR, Kim KY, Lee SH, Kim MY, Park HJ \& Jeong HS 2012 Antioxidant and antitumor activities of methanolic extracts from Humulus japonicus. Korean Journal of Food and Nutrition 25 357-361. (https:// doi.org/10.9799/ksfan.2012.25.2.357)

Lim S, Honek J, Xue Y, Seki T, Cao Z, Andersson P, Yang X, Hosaka K \& Cao Y 2012 Cold-induced activation of brown adipose tissue and adipose angiogenesis in mice. Nature Protocols 7 606-615. (https:// doi.org/10.1038/nprot.2012.013)

Lim H, Noh JR, Kim YH, Hwang JH, Kim KS, Choi DH, Go MJ, Han SS, Oh WK \& Lee CH 2016 Anti-atherogenic effect of Humulus japonicus in apolipoprotein E-deficient mice. International Journal of Molecular Medicine 38 1101-1110. (https://doi.org/10.3892/ ijmm.2016.2727)

Mangelsdorf DJ, Thummel C, Beato M, Herrlich P, Schutz G, Umesono K, Blumberg B, Kastner P, Mark M, Chambon P, et al. 1995 The nuclear receptor superfamily: the second decade. Cell $\mathbf{8 3}$ 835-839. (https://doi.org/10.1016/0092-8674(95)90199-X)

Mihaylova MM \& Shaw RJ 2011 The AMPK signalling pathway coordinates cell growth, autophagy and metabolism. Nature Cell Biology 13 1016-1023. (https://doi.org/10.1038/ncb2329)

Morrison S \& McGee SL 2015 3T3-L1 adipocytes display phenotypic characteristics of multiple adipocyte lineages. Adipocyte 4 295-302. (https://doi.org/10.1080/21623945.2015.1040612)

Park SW, Woo CJ \& Chung SK 1994 Antimicrobial and antioxidative activities of solvent fraction from Humulus japonicus. Korean Society of Food Science and Technology 26 464-470.

Park SW, Chung SK \& Kim SH 1995 Antimutagenic effects and isolation of flavonoids from Humulus japonicus extracts. Korean Society of Food Science and Technology 27 897-901.
Qiang L, Wang L, Kon N, Zhao W, Lee S, Zhang Y, Rosenbaum M, Zhao Y, Gu W, Farmer SR, et al. 2012 Brown remodeling of white adipose tissue by SirT1-dependent deacetylation of PPARgamma. Cellule 150 620-632.

Reilly SM \& Lee CH 2008 PPAR delta as a therapeutic target in metabolic disease. FEBS Letters 582 26-31. (https://doi.org/10.1016/j. febslet.2007.11.040)

Roberts CK \& Sindhu KK 2009 Oxidative stress and metabolic syndrome. Life Sciences 84 705-712. (https://doi.org/10.1016/j. lfs.2009.02.026)

Roberts LD, Bostrom P, O'Sullivan JF, Schinzel RT, Lewis GD, Dejam A, Lee YK, Palma MJ, Calhoun S, Georgiadi A, et al. 2014 Betaaminoisobutyric acid induces browning of white fat and hepatic beta-oxidation and is inversely correlated with cardiometabolic risk factors. Cell Metabolism 19 96-108. (https://doi.org/10.1016/j. cmet.2013.12.003)

Tseng YH, Cypess AM \& Kahn CR 2010 Cellular bioenergetics as a target for obesity therapy. Nature Reviews: Drug Discovery 9 465-482. (https://doi.org/10.1038/nrd3138)

Wakil SJ 1989 Fatty acid synthase, a proficient multifunctional enzyme Biochemistry 28 4523-4530. (https://doi.org/10.1021/bi00437a001)

Xu P, Wang H, Kayoumu A, Wang M, Huang W \& Liu G 2015 Diet rich in docosahexaenoic acid/eicosapentaenoic acid robustly ameliorates hepatic steatosis and insulin resistance in seipin deficient lipodystrophy mice. Nutrition and Metabolism 12 58. (https://doi. org/10.1186/s12986-015-0054-x)

Yamaguchi J, Tanaka T, Saito H, Nomura S, Aburatani H, Waki H, Kadowaki T \& Nangaku M 2017 Echinomycin inhibits adipogenesis in 3T3-L1 cells in a HIF-independent manner. Scientific Reports 7 6516. (https://doi.org/10.1038/s41598-017-06761-4)

Yu YH, Wang PH, Cheng WT, Mersmann HJ, Wu SC \& Ding ST 2010 Porcine peroxisome proliferator-activated receptor delta mediates the lipolytic effects of dietary fish oil to reduce body fat deposition. Journal of Animal Science $\mathbf{8 8}$ 2009-2018. (https://doi.org/10.2527/ jas.2009-2597)

Zhang BB, Zhou G \& Li C 2009 AMPK: an emerging drug target for diabetes and the metabolic syndrome. Cell Metabolism 9 407-416. (https://doi.org/10.1016/j.cmet.2009.03.012)

Zhang Y, Li R, Meng Y, Li S, Donelan W, Zhao Y, Qi L, Zhang M, Wang X, Cui T, et al. 2014a Irisin stimulates browning of white adipocytes through mitogen-activated protein kinase p38 MAP kinase and ERK MAP kinase signaling. Diabetes 63 514-525. (https:// doi.org/10.2337/db13-1106)

Zhang Z, Zhang H, Li B, Meng X, Wang J, Zhang Y, Yao S, Ma Q, Jin L, Yang J, et al. 2014b Berberine activates thermogenesis in white and brown adipose tissue. Nature Communications 5 5493. (https://doi. org/10.1038/ncomms6493)

Zhou Y, Wang D, Zhu Q, Gao X, Yang S, Xu A \& Wu D 2009 Inhibitory effects of A-769662, a novel activator of AMP-activated protein kinase, on 3T3-L1 adipogenesis. Biological and Pharmaceutical Bulletin 32 993-998. (https://doi.org/10.1248/bpb.32.993)

Received in final form 27 March 2019

Accepted 11 April 2019

Accepted Preprint published online 11 April 2019
(C) 2019 Society for Endocrinology Published by Bioscientifica Ltd. Printed in Great Britain 